\title{
Development of sensitivity to visual motion in macaque monkeys
}

\author{
LYNNE KIORPES AND J. ANTHONY MOVSHON
}

Center for Neural Science, New York University, New York

(Received May 24, 2004; Accepted August 31, 2004)

\begin{abstract}
The development of spatial vision is relatively well documented in human and nonhuman primates. However, little is known about the development of sensitivity to motion. We measured the development of sensitivity to direction of motion, and the relationship between motion and contrast sensitivity in macaque monkeys as a function of age. Monkeys (Macaca nemestrina, aged between 10 days and 3 years) discriminated direction of motion in random-dot kinematograms. The youngest monkeys showed directionally selective orienting and the ability to integrate motion signals at large dot displacements and fast speeds. With age, coherence sensitivity improved for all spatial and temporal dot displacements tested. The temporal interval between the dots was far less important than the spatial offset in determining the animals' performance at all but the youngest ages. Motion sensitivity improved well beyond the end of the first postnatal year, when mid-spatial-frequency contrast sensitivity reached asymptote, and continued for at least 3 years. Sensitivity to contrast at high spatial frequencies also continued to develop beyond the end of the first year. We conclude that the development of motion sensitivity depends on mechanisms beyond the low-level filters presumed to limit acuity and contrast sensitivity, and most likely reflects the function of extrastriate visual areas.
\end{abstract}

Keywords: Visual development, Visual motion, Contrast sensitivity

\section{Introduction}

A striking feature of the development of vision in primates is that different visual functions develop at different rates and have different critical periods (see, e.g. Harwerth et al., 1986; Daw, 1998). The basic elements of spatial vision-acuity and spatial contrast sensitivity - develop to adult levels over the course of the first 5-7 years after birth in humans (Mayer \& Dobson, 1982; Bradley \& Freeman, 1982; Hainline \& Abramov, 1997; Ellemberg et al., 1999) and the first year in macaque monkeys (Boothe et al., 1988; Kiorpes, 1992). More complex perceptual abilities requiring integration of information over space have more extended developmental time courses. For example, children younger than about age 3 are unable to perform a contour integration task, and their performance continues to improve into the teenage years (Pennefather et al., 1999; Kovács et al., 1999). Similarly, monkeys are unable to perform contour integration before 5-6 months and continue to improve into at least the second postnatal year (Kiorpes \& Bassin, 2003). Temporal and spatio-temporal aspects of vision also show differential development in human infants. Regal (1981) reported adult-level critical flicker fusion frequency (CFF) in 2-month-old infants (but see also, Rasengane et al., 1997). However, temporal contrast sensitivity is quite immature below the

Address correspondence and reprint requests to: Lynne Kiorpes, Center for Neural Science, 4 Washington Place, Room 809, New York, NY 10003, USA. E-mail: lynne@cns.nyu.edu
CFF up to at least 8 months (Swanson \& Birch, 1990; Teller et al., 1992; Hartmann \& Banks, 1992; Rasengane et al., 1997; Dobkins et al., 1999), though it reaches adult levels relatively early compared to spatial contrast sensitivity (Ellemberg et al., 1999). No data are as yet available on the development of temporal mechanisms in the monkey.

Some aspects of sensitivity to visual motion are evident in young infants. Reflexive eye movements $(\mathrm{OKN})$ to motion stimuli are identifiable in newborns (Volkman \& Dobson, 1976; Atkinson, 1979; Naegele \& Held, 1982; Mason et al., 2003). Psychophysical studies provide evidence for directional motion sensitivity within the first 3 months of life in humans (Braddick, 1993; Wattam-Bell, 1996; Dobkins \& Teller, 1996; Banton et al., 2001; see Braddick et al., 2003). But the age at which motion sensitivity reaches maturity is unknown.

In considering the literature on motion development, it is important to distinguish low-level mechanisms that provide local motion signals from presumptive higher level mechanisms that integrate motion information across space and time. Those tasks that show the most precocious development seem to be the ones that require only local processing. Tasks requiring integration of information may have a more prolonged developmental course. For example, Mason et al. (2003) reported that thresholds for the detection of coherent motion were not yet adult-like at 27 weeks in humans. They used a pair of large random-dot motion stimuli in a preferential-looking paradigm. One member of the pair contained a horizontal segment in which motion direction was opposite that 
in all other portions of the display. Infants at 9 weeks were able to identify the side containing the oppositely moving segment, but their thresholds were extremely high. Performance improved over the age range tested, but at the oldest test age thresholds were still higher than adults on the same task. Similarly, Hamer and Norcia (1994) showed that oscillatory motion thresholds, as measured by the visually evoked potential (VEP), are still quite immature at the end of the first postnatal year and lag behind the development of contrast thresholds. And, Giaschi and Regan (1997) report that children develop the ability to identify luminance-defined form (letters) earlier than they can process motion-defined form.

Work with monkeys suggests that sensitivity to coherent motion in extended random-dot patterns - "global" motion sensitivitydepends on the function of areas such as MT/V5 and MST, downstream of the primary visual cortex (Newsome \& Pare, 1988; Britten et al., 1992; Celebrini \& Newsome, 1995). But little is known about the development of motion sensitivity in monkeys. Mikami and Fujita (1992) measured speed thresholds between birth and 14 weeks. They found these thresholds to be quite poor in newborns, and substantially immature at the oldest age they tested. Within this limited period, Mikami and Fujita also compared the rate of change of speed sensitivity with that of acuity (as measured by other studies from the literature), and concluded that motion sensitivity develops over a slower time course than acuity. Projecting their measured motion time course forward, they suggested - on the basis of extensive extrapolation-that speed thresholds should become mature by about 3 years.

The developmental program for motion sensitivity is thus much less well documented than that for visual acuity and contrast sensitivity. Also, the relationship between motion development and spatial vision development is not known-does motion sensitivity develop in lock-step with other kinds of sensitivity, or does it have a different time course? The answer to this question determines whether theories of development need to postulate more than one mechanism limiting sensitivity to motion and form. To shed light on these issues, we have now fully characterized the development of global motion sensitivity in macaque monkeys, and have also measured the development of spatial vision in the same individuals. The time course for the development of motion sensitivity is quite extended, so that this function is not fully mature until about 3 years postnatal. This is substantially slower than the development of spatial contrast sensitivity, and suggests that the limits on global motion sensitivity during development are set by the maturation of mechanisms that lie downstream of those responsible for elementary visual discriminations.

\section{Materials and methods}

Subjects in this study were 15 visually normal Macaca nemestrina monkeys, ranging in age from 10 days to 3 years. All animals were born at the Washington National Primate Research Center, and were hand-reared in the Visual Neuroscience Laboratory at New York University. All animal care conformed to guidelines approved by the New York University UAWC and the NIH Guide for Care and Use of Laboratory Animals. The visual environment was a normal laboratory environment, which was enriched with a wide variety of appropriate visual and tactile stimuli. The animals were also given daily opportunities for interaction with other monkeys and humans.

Stimuli were presented on a Nanao T660i subtending $40 \times$ $30 \mathrm{deg}$ at $40 \mathrm{~cm}$, the viewing distance used for the youngest infants. Viewing distance was increased as the animals grew.
Typically, animals older than 6 months were tested at a distance of $100 \mathrm{~cm}$, where the monitor subtended $16 \mathrm{deg}$. For random-dot displays, the background luminance was $0.3 \mathrm{~cd} / \mathrm{m}^{2}$, and the dot luminance was $112 \mathrm{~cd} / \mathrm{m}^{2}$. For gratings, the mean luminance was $56 \mathrm{~cd} / \mathrm{m}^{2}$. Stimulus presentation was controlled by a PC computer via an ATVista graphics board (Truevision).

The motion stimuli were random-dot kinematograms, conceptually similar to those devised by Morgan and Ward (1980) but implemented as described by Britten et al. (1992). The kinematograms were presented inside a square field in the center of the display; this field subtended between 7.7 and $18.8 \mathrm{deg}$, depending on viewing distance. Each frame of this stimulus contained a fixed number of dots that changed position from frame to frame. Any given dot at time $\mathrm{t}$ appeared at a location $(x, y)$. At time $t+\Delta t$, with a probability that we term the coherence, the dot reappeared at location $(x+\Delta x, y)$. The coherence determined the strength of the global motion signal. If the dot did not reappear, it was replaced by a new dot at a random location. The total number of dots was the same on each frame. The number of appearances of any given dot was related to the coherence. For instance, if the coherence was 0.2 , the probability of a dot reappearing once was 0.2 , twice was 0.04 , and three times was 0.008 . Because dot lifetime was limited, it was difficult to judge direction by tracking any single dot or a group of dots (except, of course, when the coherence approached 1). The speed of coherent motion was $\Delta x / \Delta t$, while the incoherent dots provided a masking motion background whose speeds and directions were essentially uniform in distribution.

Behavioral methods were similar to those described elsewhere. We used a combination of preferential looking (in animals younger than about 10 weeks) (Kiorpes, 1992) and standard operant conditioning techniques (Kiorpes et al., 1993; Kiorpes \& Movshon, 1998) to collect data from the developing animals. The psychophysical task was a two-alternative forced-choice directional discrimination. A rectangular field of random dots appeared in the center of the display screen; coherent motion was translational, either rightward or leftward. The size of the display, and of the dots themselves, was increased for the youngest ages. In the case of preferential looking the human observer was required to judge the direction of motion of the dots, left or right, based on the looking behavior or eye movements of the infant-OKN was the most reliable cue. In the case of operant testing, the subject signalled the direction of motion with a bar pull; a squirt of age appropriate liquid (infant formula or apple juice) was delivered following correct responses.

We measured coherence threshold by varying the strength of the motion signal across trials using method of constant stimuli. We fit psychometric functions, based on 3-5 coherence levels, and at least 60 trials per level, for each of a range of $\Delta x$ and $\Delta t$ values. Data collection was counterbalanced across $\Delta x$ for a given $\Delta t$, and across $\Delta t$. Threshold estimates ( $75 \%$ correct) and associated standard errors were calculated using Probit analysis (Finney, 1971) of the log-transformed data sets. We took motion sensitivity to be the inverse of coherence at threshold.

To compare the development of motion sensitivity with basic spatial vision, we measured either grating acuity or spatial contrastsensitivity functions. We assessed the status of spatial visual development immediately before or at the conclusion of motion testing at each age. Acuity and contrast sensitivity were measured using conventional methods (Kiorpes, 1992; Kiorpes et al., 1993). Square-wave or sinusoidal gratings were generated under computer control with the same video display system as was used for 
motion testing. Grating patches were vignetted by a two-dimensional spatial Gaussian $(\sigma=0.75 \mathrm{deg}$, except for very low spatial frequencies for which $\sigma$ was increased to keep at least three grating cycles visible). Spatial frequencies ranged from 0.3 cycles/ deg to 16 cycle/deg; viewing distance ranged from $0.3 \mathrm{~m}$ to $2 \mathrm{~m}$. The monkey's task was to detect the presence of the grating patch on either the right or left side of an otherwise homogeneous gray field that matched the grating in space-average luminance. Threshold values and standard errors of estimate were obtained by Probit analysis of the log-transformed data sets (Finney, 1971) using a maximum-likelihood technique.

We used an iterative method to fit a double-exponential function (Wilson \& Bergen, 1979; Kiorpes et al., 1993; Kiorpes \& Kiper, 1996; Kozma \& Kiorpes, 2003) to both motion and contrastsensitivity data, and extracted the value of peak sensitivity and its location on the abscissa from the fits.

\section{Results}

The youngest monkeys we tested, in the range 10-20 days, were able to indicate direction of motion in the random-dot displays. Using forced-choice preferential looking, a human observer could reliably determine direction of motion using either fixation behavior of the infant or OKN. However, motion discrimination was apparent only with large dot displacements and a short temporal offset $(\Delta t)$ (i.e. at fast dot speeds). Representative developmental data from an individual monkey tested longitudinally are shown in Fig. 1. Motion sensitivity is plotted as a function of dot displacement (the related dot speed axis is plotted at the top of the panel) for monkey JU at four ages. At the youngest age, 2 weeks, she was

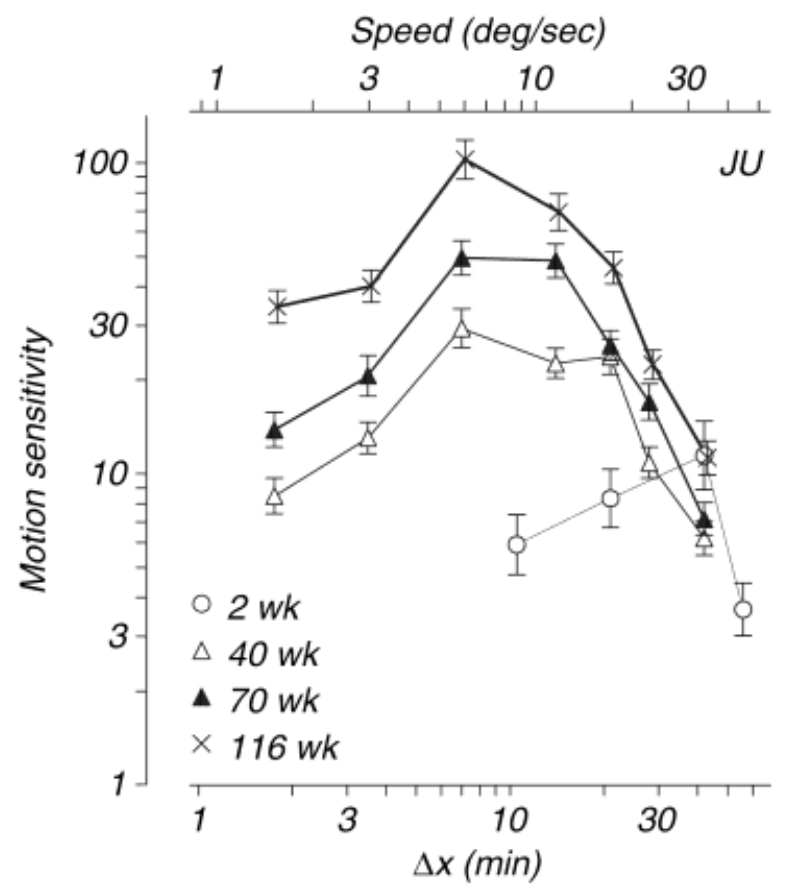

Fig. 1. Development of sensitivity to random-dot motion in a single infant monkey, tested at four ages from 2 weeks to 116 weeks. The abscissae provide scales of $\Delta x$ (interdot spacing) and speed. $\Delta t$ (temporal interdot interval) was $19 \mathrm{~ms}$. Motion sensitivity is the inverse of the motion coherence at threshold. sensitive to dot displacements $(\Delta x)$ in the range of 10-60 min. With age, her sensitivity increased 10 -fold while the range of sensitivity expanded to include finer dot displacements. Close examination of the data shows that within the first postnatal year, the range of $\Delta x$ at which she could discriminate motion direction expanded to comprise the smallest displacements tested, however, overall sensitivity was not fully developed at that age. The most surprising finding was that sensitivity continued to improve well beyond the end of the first postnatal year (70 and 116-week data sets), and in fact increased up to 3 years of age.

The data shown in Fig. 1 were collected at a temporal offset of $19 \mathrm{~ms}$, which translates to relatively fast speeds. To cover a wider range of dot speeds, we measured motion sensitivity at two longer temporal offsets, 37 and 56 ms. Fig. 2 shows motion sensitivity for another monkey (OJ) tested longitudinally plotted as a function of $\Delta t$ at three dot displacements ranging from coarse to fine. Motion sensitivity was generally best for a $\Delta t$ of $19 \mathrm{~ms}$, and fell slightly as $\Delta t$ increased. This fall was most marked at younger ages. At 3 weeks, OJ's motion discrimination was measurable only at a $\Delta t$ of 19 ms. By 14 weeks, she was able to integrate over a 37-ms temporal offset and was able to use a $\Delta t$ of $56 \mathrm{~ms}$ by 23 weeks. As noted above, young monkeys were not able to discriminate motion direction with fine $\Delta x$, thus there are no data at 3 week for a $\Delta x$ of 4 min (Fig. 2C). At the oldest age, motion sensitivity was less affected by $\Delta t$ than it had been earlier, but the changes in sensitivity to temporal offset during development were much less marked than the changes in sensitivity to spatial offset shown in Fig. 1.

The time course of development of motion sensitivity, based on data from the entire population of animals tested, is shown in Fig. 3. Motion sensitivity, the highest sensitivity at each age and $\Delta t$, for each monkey is plotted as a function of age. The data collected at ages up to 3 weeks were obtained with preferential looking, while at older ages data were obtained with operant techniques (see Methods). There was a steady, slow increase of sensitivity up to about 3 years of age; the developmental profile is similar for all three values of $\Delta t$, as indicated by the different colored symbols in Fig. 3.

The data in Figs. 2 and 3 suggest that temporal offset exerts a weak but consistent effect on sensitivity at all ages and ranges of displacement; the curves decrease monotonically but gently with $\Delta t$. On the other hand, it is clear from Fig. 1 that spatial displacement is an important determinant of sensitivity. Another possibility is that it is neither $\Delta x$ nor $\Delta t$ alone that determine sensitivity, but their ratio, the dot speed. To directly evaluate the effect of dot speed, as opposed to dot displacement, on motion sensitivity we plot a complete data set against both parameters in Fig. 4. Data from JU, measured at 40 weeks, are plotted as a function of dot speed in Fig. 4A. Clearly, the functions are shifted and sensitivity varies over a wide range for a given speed (e.g. $10 \mathrm{deg} / \mathrm{s}$ ) depending on the temporal offset used. The same data set is plotted as a function of dot displacement in Fig. 4B. The functions are more similar in position when plotted as a function of $\Delta x$, although there is a small reduction of sensitivity at the longest $\Delta t$ (as also seen in Fig. 2). This result suggests that motion sensitivity is, in fact, more dependent on dot displacement than on dot speed. If so, then peak displacement, the $\Delta x$ at which motion sensitivity was highest, should be similar at each age regardless of $\Delta t$.

Fig. 5 shows the change in optimal $\Delta x$ as a function of age for all three values of $\Delta t$. The steady shift to finer $\Delta x$ with age is essentially independent of $\Delta t$, confirming that the spatial parameters of the dot display are much more important determinants of 

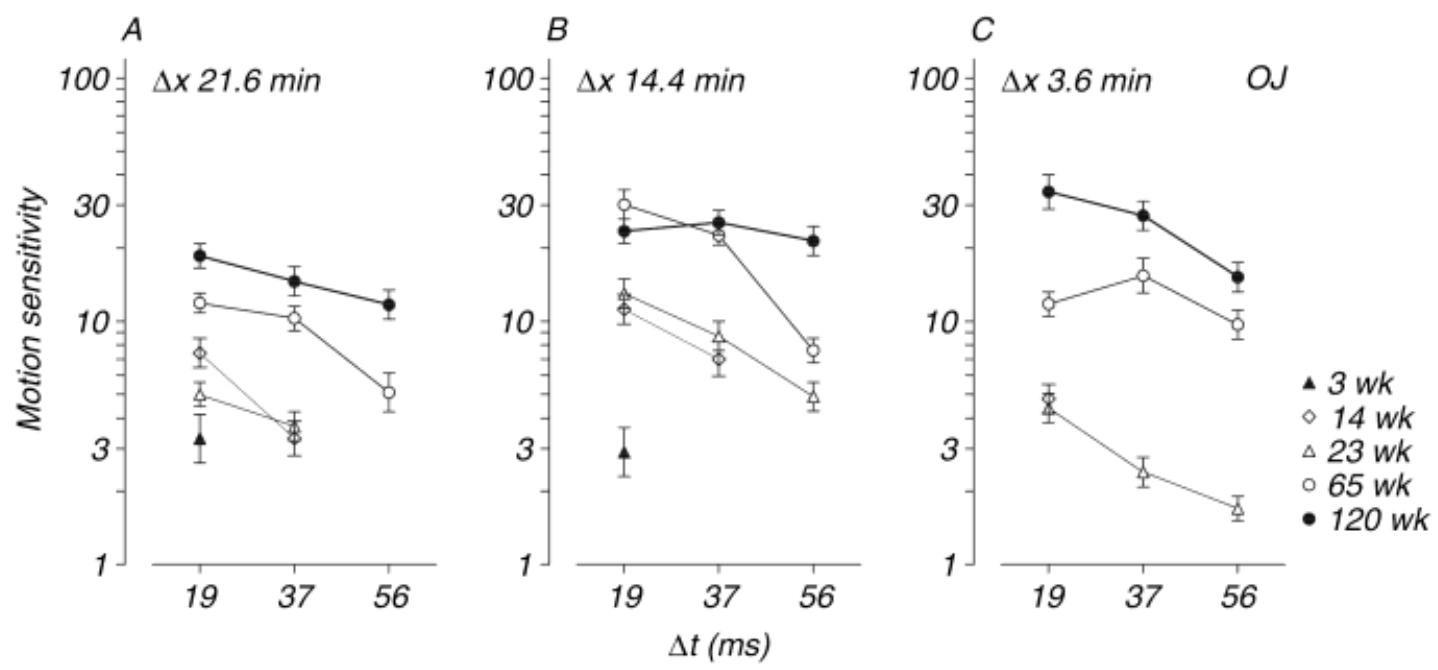

Fig. 2. Effect of the temporal interdot interval on the development of motion sensitivity in a single infant monkey, tested at five ages from 3 weeks to 120 weeks. Data are shown for three values of $\Delta x: 21.6,14.4$, and 3.6 min (A-C).

sensitivity in this task than are temporal parameters. Developmentally, then, we should expect to find a parallel between animals' ability to resolve spatial detail and discriminate motion. To explore this relationship, we used motion-sensitivity and contrast-sensitivity data collected from individual animals at comparable ages to extract spatial parameters of both functions. We collected data for six spatial frequencies ranging from 0.5 cycles/deg to 16 cycles/ $\mathrm{deg}$, and seven dot displacement values ranging from $1.7 \mathrm{~min}$ to 43 min, across animals and ages.

There is no absolute basis for comparison between the spatialfrequency dependence of contrast sensitivity and the spatial-offset dependence of motion sensitivity. For grating detection, it is

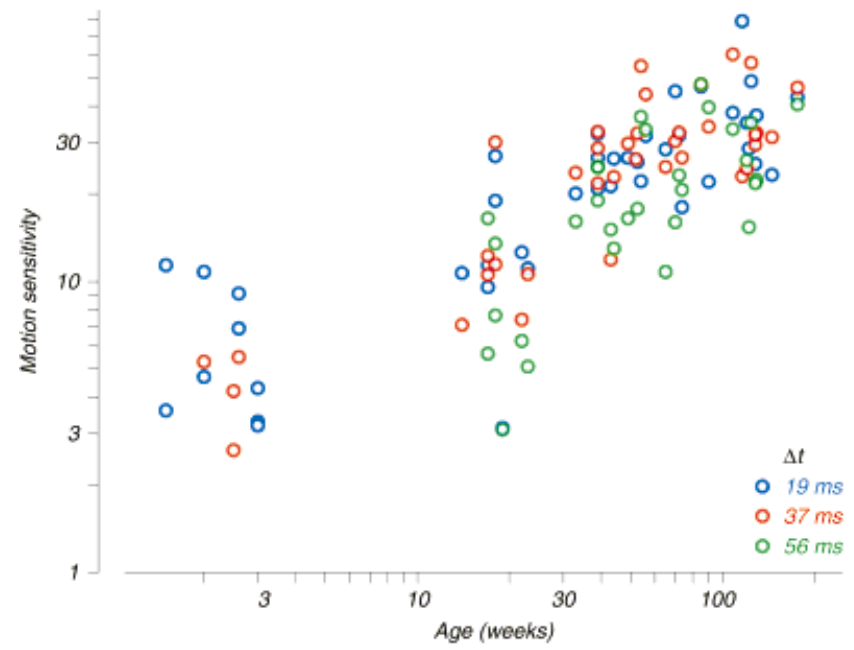

Fig. 3. Developmental progression of the sensitivity value at the peak of the motion-sensitivity function, shown for all three tested values of $\Delta t$. The peak sensitivity was extracted, in most cases, by fitting a suitable function to data like those shown in Fig. 1 (see text for details); in the case of some infants that had a restricted range of sensitivity, we took the best sensitivity achieved at any $\Delta x$. reasonable to assume that mechanisms tuned to the test frequency are responsible for detection. But the directional information in a random-dot display is present at a wide range of spatial frequencies, and can in principle be analyzed by mechanisms sensitive to any informative spatial frequency. Smith et al. (2002) analyzed the responses of real and simulated neurons to dynamic Glass patterns (akin to our kinematograms except with a $\Delta t$ of 0 ). They concluded that neuronal responses were most informative when $\Delta x$ corresponded to roughly half the wavelength of the optimal spatial frequency. For comparison purposes, we therefore take $2 / \Delta x$ as the "equivalent spatial frequency" of a kinematogram.

Fig. 6 shows contrast sensitivity (green) and motion sensitivity (red) as a function of age for five spatial scales (coarse to fine from top to bottom of the figure) for which we had data at matched true and equivalent spatial frequencies. We fit the time course of development for each spatial frequency or $\Delta x$ with a suitable saturating function (Michaelis-Menten) to describe the gradual approach to adult values of sensitivity with age at each spatial scale. Consistent with previous reports (Boothe et al., 1988; Movshon \& Kiorpes, 1988), there was little change in contrast sensitivity with age at the low spatial frequency (1 cycle/deg, Fig. 6A, green), just as there was little improvement in motion sensitivity at the matched $\Delta x$ (29 min, Fig. 6A, red). Contrast sensitivity at midrange frequencies (2-4 cycles/deg, Figs. 6B \& 6C, green) improved substantially with age, reaching asymptotic levels by about 40 weeks. On the other hand, motion sensitivity at matched $\Delta x$ (14.4 and $7.2 \mathrm{~min}$, Figs. 6B \& 6C, red) showed steady improvement well beyond this age, and no saturation is evident even up to the oldest age tested. Interestingly, sensitivity development at finer spatial scales had a similar course for motion and contrast (Figs. 6D \& 6E), showing continued improvement well beyond the end of the first year. Comparisons across the curves in Fig. 6 show that while contrast sensitivity and motion sensitivity generally increase more at fine than coarse spatial scales, the developmental profiles for these two functions differ in detail, specifically at their points of asymptote. For the spatial scales where sensitivity reaches its highest level on both measures, there was substantial development of motion sensitivity even after contrast sensitivity became fully adult. 


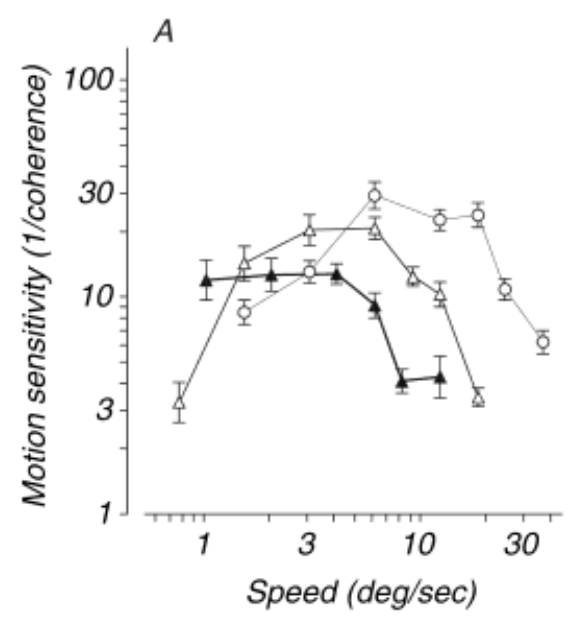

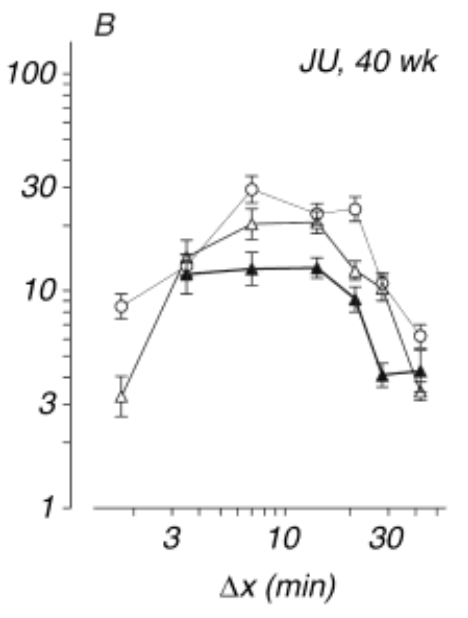

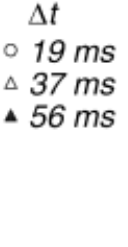

Fig. 4. Dependence of motion sensitivity on $\Delta x$ and speed. The same family of curves, obtained from a single monkey at the age of 40 weeks using three different values of $\Delta t$, are plotted as a function of speed (A) and $\Delta x(\mathrm{~B})$.
When considering the plots in Fig. 6, it is important to remember that there is no principled relationship between the absolute values of motion and contrast sensitivity. That is, even if they depended on exactly the same mechanisms, the values would not necessarily change proportionally across age and scale. It is therefore the differences in the shape of the functions rather than their fine structure that are most revealing of the similarities and differences in the underlying developmental events. So it is not necessarily meaningful that the motion- and contrast-sensitivity curves have different slopes in some panels, but it is important that relationship between motion sensitivity and contrast sensitivity changes with spatial scale as well as age. This is most obvious from the fact that motion-sensitivity curves lie below the contrastsensitivity curves in some panels while the situation is reversed in others.

\section{Discussion}

Infant monkeys are sensitive to direction of motion as early as 10 days after birth, and can use global motion information to control behavior. The range of threshold variation for these young infants was quite large, and overlapped that of the animals tested between 10 and 20 weeks with operant methods (Fig. 3).We used

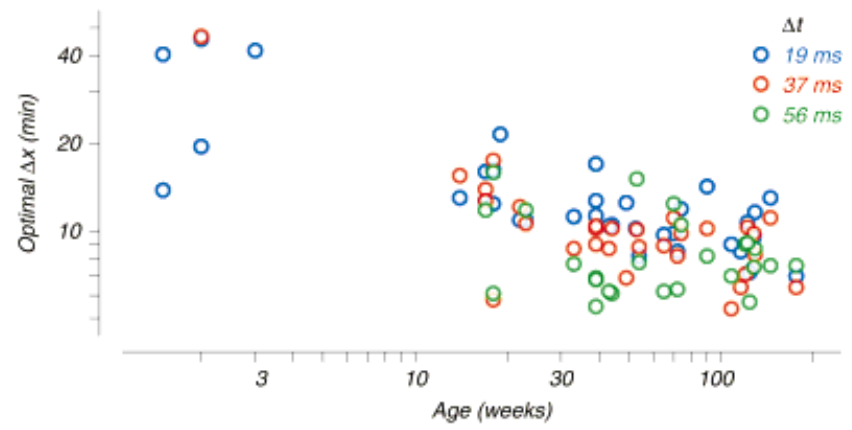

Fig. 5. Developmental progression of the value of $\Delta x$ at the peak of the motion sensitivity function, shown for all three tested values of $\Delta t$. The optimal value of $\Delta x$ was extracted by fitting a suitable function to data like those shown in Fig. 1 (see text for details). Cases were excluded in the event that the range of sensitivity was too limited to fit the data with the function. a preferential-looking paradigm to test infants at the very young ages. The experimenter used a variety of cues, including tracking movements, OKN, or fixation preference, to make her judgments, depending on what was most reliable for a given infant. Mason et al. (2003) have reported that OKN measurements reveal earlier abilities and lower thresholds for global motion discrimination than traditional fixation preference measurements in human infants, and it might be that the early measurements based on OKN slightly overestimate motion sensitivity. Thus, the large variance of thresholds at this age could be due to the mixture of cues used by the observer. However, it could also result from individual variation in developmental rates. Nonetheless, it is clear that sensitivity to global motion is evident in the early postnatal weeks and develops slowly and steadily thereafter.

Best coherence thresholds at the youngest ages were between 0.2 and 0.3 , and decreased to 0.01 to 0.03 over the subsequent 3 years. The extent of development in monkeys, about a factor of 10 , was similar to that reported in humans (Wattam-Bell, 1994). Coherence thresholds in 3-month-old human infants, under his conditions of test, were about 0.5 , and decreased to around 0.05 in adults. The difference in adult levels of performance between monkeys and humans could be due to task differences, though we have often found monkeys' performance on motion-detection tasks to be superior to humans' (unpublished observations). The long, slow time course of motion-sensitivity development that we found is consistent with a study in humans showing continued improvement in coherence threshold up to about age 10 (Gunn et al., 2002).

\section{Determinants of sensitivity to speed}

In this study, we explored direction discrimination over a wide range of dot displacements at three different temporal offsets. The combination of spatial and temporal offsets covered a range of speeds from $0.8 \mathrm{deg} / \mathrm{s}$ to $40 \mathrm{deg} / \mathrm{s}$. We found little evidence that performance depended on dot speed. Motion sensitivity, when characterized in terms of dot speed, varied greatly depending on the underlying temporal offset used (Fig. 4). This is consistent with prior reports by Braddick and others who concluded that displacement rather than speed was the more important factor for performance with apparent motion displays such as ours (Baker \& Braddick, 1985; see Braddick et al., 2003). By separately analyzing the contribution of temporal and spatial factors, we found a strong dependence of sensitivity on dot displacement at all ages. 


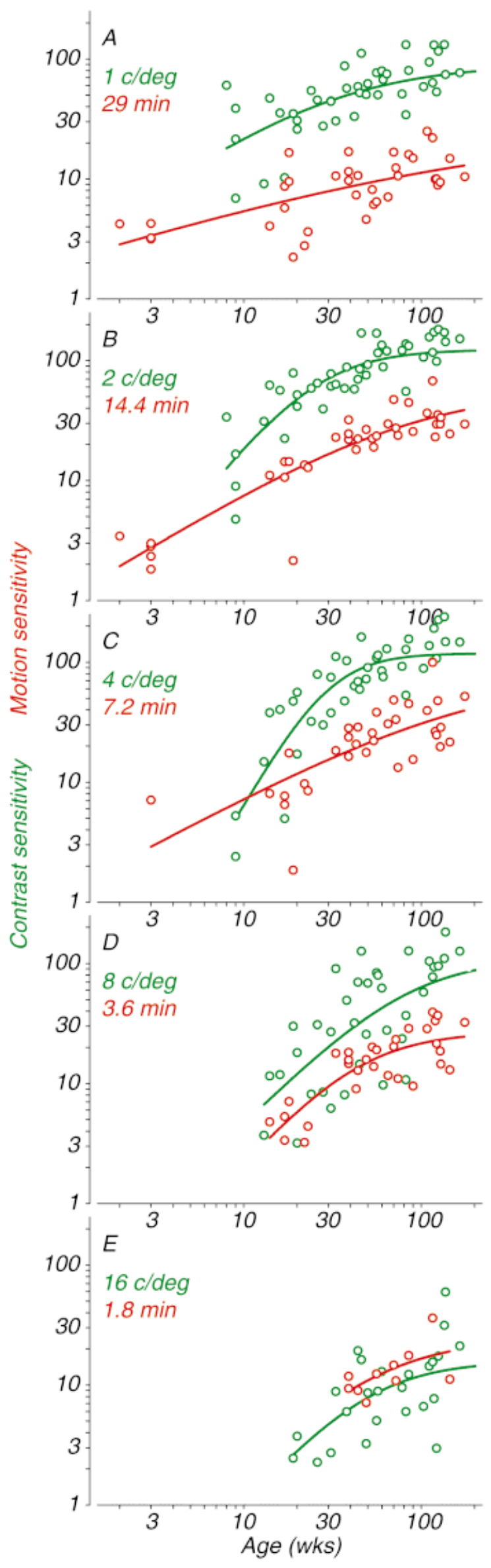

It is noteworthy that the youngest monkeys were unable to extract the motion signal at the longest $\Delta t, 56 \mathrm{~ms}$. This result, coupled with the unsurprising finding that the young infants could not resolve fine-scale dot displacements, means that the young monkeys are particularly poor at discriminating slow speeds. Thus development proceeded from fast to slow speeds following the well-known development of contrast sensitivity from coarse-tofine scale. This pattern of development seems contrary to that reported by Wattam-Bell in human infants (Wattam-Bell, 1991, 1992, 1996). He used VEP and behavioral methods (preferential looking) to characterize the onset of directional motion sensitivity between 8 and 16 weeks. Wattam-Bell characterized the development of motion sensitivity by tracking $V_{\min }$ and $V_{\max }$, the minimum and maximum speeds that infants could discriminate; he found both increases in $V_{\max }$ and decreases in $V_{\min }$. This is akin to measuring the low- and high-cutoff values of $\Delta x$ in our study. Inspection of our longitudinal data (Figs. 1, 2, \& 6) shows that our monkeys' sensitivities improved at both extremes of the speed range, in general agreement with the findings in humans. But this change is in large part due to the increase in motion sensitivity with age, which causes both shoulders of the sensitivity curve to rise along with its middle. Wattam-Bell emphasizes the changes in $V_{\max }$ in his experiments, which show an increase in the high-speed cutoff during development. But if one measures only a single or small selected range of conditions, the pattern of sensitivity change with age will depend on precisely where one chooses to sample in $\Delta x / \Delta t$ space. What is missed by characterizing only $V_{\min }$ or $V_{\max }$ is the shift in optimal values for motion detection (Fig. 5), which very clearly proceeds from larger to smaller values of $\Delta x$, from higher to lower speeds. Consistent with our findings, Banton and Bertenthal (1996) found better performance for faster speeds with a uniform motion display and no change in overall sensitivity over an age range comparable to that studied by Wattam-Bell. Also, several other studies have reported substantial improvements in sensitivity to slow speeds over the range 2-6 months (Aslin \& Shea, 1990; Finlay et al., 1991, Roessler \& Dannemiller, 1997).

\section{Spatial characteristics of motion and contrast detection}

From our results, it is clear that $\Delta x$, not speed, is the main determinant of motion-discrimination performance throughout development. To make a direct comparison of the spatial properties of contrast- and motion-sensitivity during development, we created interpolated contrast and motion sensitivity functions from the functions fit to the data sets shown in Fig. 6, as well as to other longitudinal data that we compiled and fitted in the same way. The resulting functions are plotted on matched axes for a range of ages in Fig. 7. Motion sensitivity (B) is plotted as a function of equivalent spatial frequency, $(\Delta x / 2$ as described above); the corresponding age in weeks is noted to the right of each function. Comparing Figs. 7A with 7B, a number of points are clear.

Fig. 6. Developmental progression of motion (red) and spatial contrast sensitivity (green) for five spatial conditions (A-E) for which grating spatial frequency matched the kinematogram "equivalent spatial frequency" $(\Delta x / 2$, see text for details), using merged data for $\Delta t$ values of 19 and $37 \mathrm{~ms}$. Each data set is described by a Michaelis-Menten function: $\operatorname{age}^{n} /\left(\operatorname{age}^{n}+\operatorname{age}_{50}^{n}\right)$, where $a g e_{50}$ is the age at which sensitivity reached half-maximum, and the exponent $n$ governs the slope of the function below age $_{50}$. 

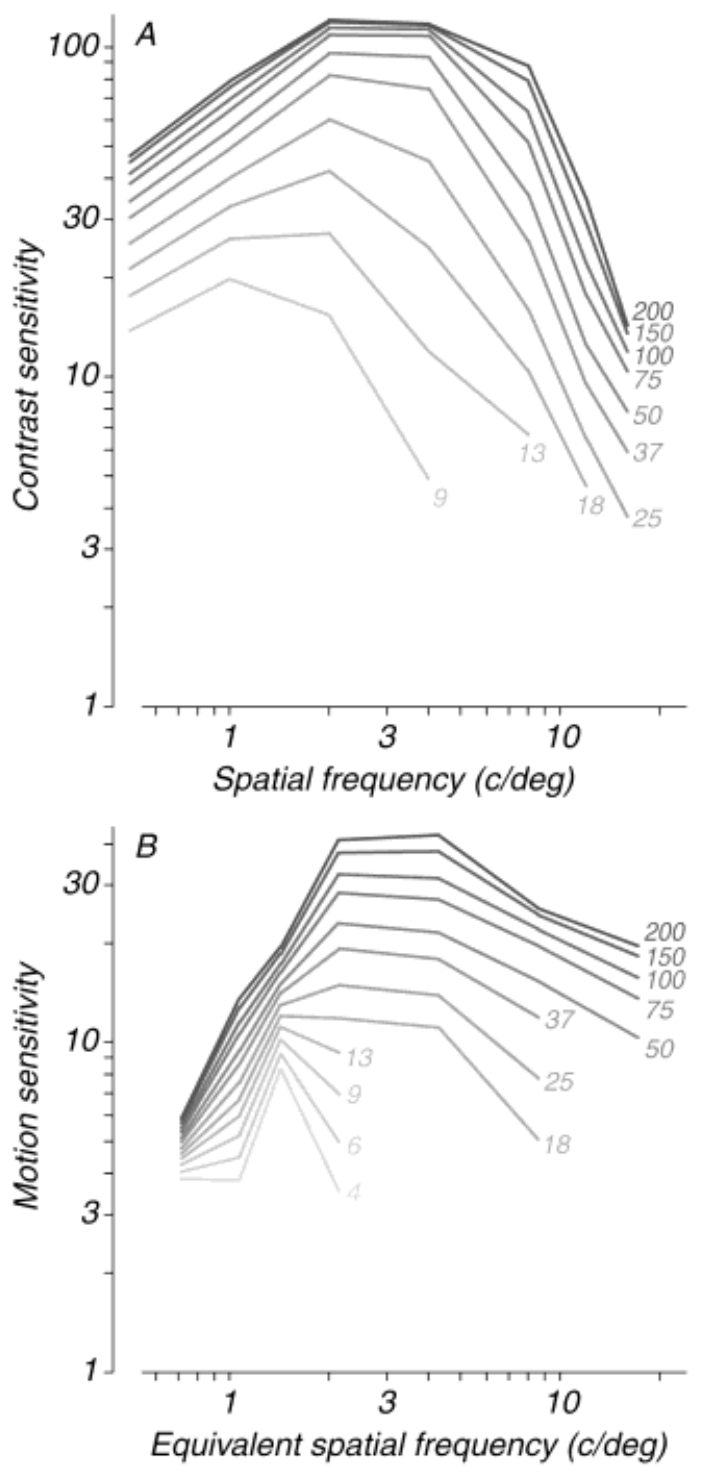

Fig. 7. Population average interpolated contrast (A) and motion (B) sensitivity functions shown for 12 ages from 4 weeks to 200 weeks. The functions were derived by picking off suitable age points from the five pairs of smooth curves shown in Fig. 6, and from three similar curves taken for other spatial frequencies and $\Delta x$ values not illustrated in Fig. 6 .

First, the shape of the function is not the same for motion and contrast sensitivity: the low-frequency loss of sensitivity is much more profound for motion than for contrast, while at high frequencies that situation is reversed. The reason for the steeper lowfrequency slope for motion detection than for contrast detection is probably that, in contrast sensitivity, low spatial-frequency detection can be aided by integrating light over large areas. In motion sensitivity, the stimulus element detected contains just the same pair of dots at all separations, and there is no benefit to integrating over larger areas; in fact, performance may suffer because the number of other dots that intrudes between the pair increases with $\Delta x$. The shallower high-frequency slope of the motion-sensitivity functions is probably due to the breakdown of our assumed "equivalent spatial frequency" relationship for small $\Delta x$. This relationship is based on the assumption that contrast sensitivity is roughly uniform across the range of interest (Smith et al., 2002). But for high spatial frequencies, the steep fall in contrast sensitivity combined with the broad-band information in the dot display will make channels preferring lower spatial frequencies relatively more sensitive, raising the high-frequency end of the motionsensitivity function.

Second, the development of sensitivity at the peak of the function ends much earlier for contrast than motion sensitivity; this reflects the flattening of the contrast-sensitivity development curves in Figs. 6B and 6C. Continued improvement in performance with age on both tasks occurs at fine spatial scales, especially in animals older than 25 weeks. Beyond 50 weeks, motion-sensitivity development appears to outstrip contrast sensitivity.

Third, in animals younger than about 20 weeks, high spatial frequencies can be detected, but the visual mechanisms responsible for processing these frequencies do not seem to support motion discrimination. So, for example, at the age of 10 weeks, the average monkey can resolve spatial frequencies in excess of 8 cycles/deg, yet motion sensitivity cannot be measured beyond an equivalent frequency of 2 cycles/deg. This suggests an immaturity of the downstream neural mechanisms responsible for extracting motion from (apparently) robust form signals.

\section{Extended development of visual sensitivity}

One surprising finding was the subtle but consistent continued development of sensitivity to high spatial frequencies beyond the end of the first postnatal year, which accompanied the extended development of motion sensitivity. Boothe et al. (1988) found the contrast sensitivity to be adult-like by the end of the first postnatal year in this species. However, they did not continue testing animals beyond that time. With the variability of sensitivity across adults that is commonly noted in humans as well as animals, it is difficult to specify the end of development with precision. Recent studies of humans have also reported continued development of acuity and contrast sensitivity into late childhood (Hainline \& Abramov, 1997; Neu \& Sireteanu, 1997; Ellemberg et al., 1999) whereas earlier studies had concluded that the process was complete by 3-5 years (Mayer \& Dobson, 1982; Birch et al., 1983). The extended development of high spatial-frequency contrast sensitivity, beyond the age at which sensitivity asymptotes at lower spatial frequencies, creates a continued gradual broadening in the shape of the contrast-sensitivity function (cf. Movshon \& Kiorpes, 1988). Since the spatial structure of receptive fields in V1 of macaque monkeys is essentially adult-like by 4 months (Chino et al., 1997; Movshon et al., 1999; Kiorpes \& Movshon, 2003), it may be that this late development of high-frequency sensitivity reflects the delayed maturation of a downstream mechanism that pools information from spatially tuned mechanisms in V1.

The long developmental time course for motion sensitivity contrasts with the finding that neurons in V1 have fully adult-like direction selectivity in newborn macaques (Chino et al., 1997; Movshon et al., 1999; Kiorpes \& Movshon, 2003). Performance on random-dot motion tasks may depend on more than just direction selectivity in V1, perhaps integrative mechanisms in or beyond area MT. We have recently found that MT neurons are fully direction selective in newborn macaques (Movshon et al., 2003). But we also found significant immaturities in both the spatial and temporal integration of motion signals, which could underlie the behavioral immaturities and extended development of directional motion discrimination reported here. 


\section{Acknowledgments}

This work was supported by NIH grants EY05864 (L.K.) and EY02017 (J.A.M.), and RR00166 to the Washington National Primate Research Center, and by an investigatorship to J.A. Movshon from the Howard Hughes Medical Institute. We thank Hannah Bayer, Nesha Burghardt, and Chao Tang for their contributions to this work, and Michael Gorman and Jeanine DiMitri D'Agostino for assistance with animal care and testing.

\section{References}

AsLIN, R.N. \& SHEA, S.L. (1990). Velocity thresholds in human infantsimplications for the perception of motion. Developmental Psychology 2, 589-598.

AtKinson, J. (1979). Development of optokinetic nystagmus in the human infant and monkey infant: An analogue to development in kittens. In NATO Advanced Study Institute Series, Developmental Neurobiology of Vision, ed. Freeman, R.D., pp. 277-287. New York: Plenum Press.

BAKER, C.L. \& BRAdDICK, O. (1985). Temporal properties of the shortrange process in apparent motion. Perception 14, 181-192.

Banton, T., Dobkins, K. \& Bertenthal, B.I. (2001). Infant direction discrimination thresholds. Vision Research 41, 1049-1056.

Birch, E.E., Gwiazda, J., Bauer, J.A., Naegele, J. \& Held, R. (1983). Visual acuity and its meridional variations in children aged 7-60 months. Vision Research 23, 1019-1024.

Boothe, R.G., Kiorpes, L., Williams, R.A. \& Teller, D.Y. (1988). Operant measurements of spatial contrast sensitivity in infant macaque monkeys during development. Vision Research 28, 387-396.

BRADDICK, O. (1993). Orientation and motion-selective mechanisms in infants. In Early Visual Development: Basic and Clinical Research, ed. Simons, K., pp. 163-177. New York: Oxford University Press.

Braddick, O., Atkinson, J. \& Wattam-Bell, J. (2003). Normal and anomalous development of visual Motion processing: motion coherence and 'dorsal-stream vulnerability'. Neuropsycologia 41, 1769-1784.

Bradley, A. \& Freeman, R.D. (1982). Contrast sensitivity in children. Vision Research 22, 953-959.

Britten, K.H., Shadlen, M.N., Newsome, W.T. \& Movshon, J.A. (1992). The analysis of visual motion: A comparison of neuronal and psychophysical performance. Journal of Neuroscience 12, 4745-4765.

Celebrini, S. \& Newsome, W.T. (1995). Microstimulation of extrastriate area MST influences performance on a direction discrimination task. Journal of Neurophysiology 73, 437-448.

Chino, Y.M., Smith, E.L., III, Hatta, S. \& Cheng, H. (1997). Postnatal development of binocular disparity sensitivity in neurons of the primate visual cortex. Journal of Neuroscience 17, 296-307.

Daw, N.W. (1998). Critical periods and amblyopia. Archives of Ophthalmology 116, 502-505.

DobKINS, K.R. \& Teller, D.Y. (1996). Infant motion:detection (M:D) ratios for chromatically defined and luminance-defined moving stimuli. Vision Research 36, 3293-3310.

Dobkins, K.R., Anderson, C.M. \& LiA, B. (1999). Infant temporal contrast sensitivity functions (tCSFs) mature earlier for luminance than for chromatic stimuli: Evidence for precocious magnocellular development? Vision Research 39, 3223-3239.

Ellemberg, D., Lewis, T.L., Liu, C.H. \& Maurer, D. (1999). Development of spatial and temporal vision during childhood. Vision Research 39, 2325-2333.

Finlay, D.C., Chorlton, M.C. \& Boulton, J.C. (1991). Motion thresholds in infants to sinusoidal gratings. Journal of General Psychology 118, 263-270.

Finney, D.J. (1971). Probit Analysis. New York: Cambridge University Press.

Giaschi, D. \& Regan, D. (1997). Development of motion-defined figureground segregation in preschool and older children, using a letteridentification task. Optometry and Vision Science 74, 761-767.

Gunn, A., Cory, E., Atkinson, J., Braddick, O., Wattam-Bell, J., Guzzetta, A. \& Cioni, G. (2002). Dorsal and ventral stream sensitivity in normal development and hemiplegia. NeuroReport 13, 843-847.

Hainline, L. \& Abramov, I. (1997). Development of spatial contrast sensitivity from infancy to adulthood: Psychophysical data. Optometry and Vision Science 74, 785-789.

Hamer, R.D. \& NorCia, A.M. (1994). The development of motion sensitivity during the first year of life. Vision Research 34, 2387-2402.
Hartmann, E.E. \& Banks, M.S. (1992). Temporal contrast sensitivity in human infants. Vision Research 32, 1163-1168.

Harwerth, R.S., Smith, E.L., III, Duncan, G.C., Crawford, M.L. \& vON NoORDEN, G.K. (1986). Multiple sensitive periods in the development of the primate visual system. Science 232, 235-238.

KIORPES, L. (1992). Development of vernier acuity and grating acuity in normally reared monkeys. Visual Neuroscience 9, 243-251.

KioRPES, L. \& KIPER, D.C. (1996). Development of contrast sensitivity across the visual field in macaque monkeys (Macaca nemestrina). Vision Research 36, 239-247.

Kiorpes, L. \& Movshon, J.A. (1998). Peripheral and central factors limiting the development of contrast sensitivity in macaque monkeys. Vision Research 38, 61-70.

Kiorpes, L. \& Movshon, J.A. (2003). Neural limitations on visual development in primates. In The Visual Neurosciences, ed. Chalupa, L.M. \& Werner, J.S., pp. 159-173. Cambridge, Massachusetts: MIT Press.

KioRPES, L. \& BAssin, S.A. (2003). Development of contour integration in macaque monkeys. Visual Neuroscience 20, 567-575.

Kiorpes, L., Kiper, D.C. \& Movshon, J.A. (1993). Contrast sensitivity and vernier acuity in amblyopic monkeys. Vision Research 33, 2301-2311.

Kovács, I., Kozma, P., Fehér, Á. \& Benedek, G. (1999). Late maturation of visual spatial integration in humans. Proceedings of the National Academy of Sciences of the U.S.A. 96, 12204-12209.

Kozma, P. \& Kiorpes, L. (2003). Contour integration in amblyopic monkeys. Visual Neuroscience 20, 577-588.

Mason, A.J.S., Braddick, O.J. \& Wattam-Bell, J. (2003). Motion coherence thresholds in infants-different tasks identify at least two distinct motion systems. Vision Research 43, 1149-1157.

Mayer, D.L. \& Dobson, V. (1982). Visual acuity development in infants and young children as assessed by operant preferential looking. Vision Research 22, 1141-1151.

Mikami, A. \& Fujita, K. (1992). Development of the ability to detect visual motion in infant macaque monkeys. Developmental Psychobiology $25,345-354$.

Morgan, M.J. \& WARD, R. (1980). Conditions for motion flow in dynamic visual noise. Vision Research 20, 431-435.

Movshon, J.A. \& Kiorpes, L. (1988). Analysis of the development of spatial contrast sensitivity in monkey and human infants. Journal of the Optical Society of America A 5, 2166-2172.

Movshon, J.A., Kiorpes, L., Cavanaugh, J.R. \& Hawken, M.J. (1999). Receptive field properties and surround interactions in V1 neurons in infant macaque monkeys. Society for Neuroscience Abstracts 25, 1048.

Movshon, J.A., Rust, N.C., Kohn, A., Kiorpes, L. \& Hawken, M.J. (2003). Receptive-field properties of MT neurons in infant macaques. Society for Neuroscience Abstracts 126.8 .

NAEgele, J.R. \& Held, R. (1982). The postnatal development of monocular optokinetic nystagmus in infants. Vision Research 22, 341-346.

Neu, B. \& Sireteanu, R. (1997). Monocular acuity in preschool children: Assessment with the Teller and Keeler acuity cards in comparison to the C-test. Strabismus 5, 185-201.

Newsome, W.T. \& Pare, E.B. (1988). A selective impairment of motion perception following lesions of the middle temporal visual area (MT). Journal of Neuroscience 8, 2201-2211.

Pennefather, P.M., Chandna, A., Kovacs, I., Polat, U. \& Norcia, A.M. (1999). Contour detection threshold: Repeatability and learning with 'contour cards'. Spatial Vision 12, 257-266.

Rasengane, T.A., Allen, D. \& Manny, R.E. (1997). Development of temporal contrast sensitivity in human infants. Vision Research 37, $1747-1754$.

Regal, D.M. (1981). Development of critical flicker fusion frequency in human infants. Vision Research 21, 549-555.

Roessler, J.S. \& Dannemiller, J.L. (1997). Changes in human infants' sensitivity to slow displacements over the first 6 months. Vision Research 37, 417-423.

Smith, M.A., BaIR, W. \& Movshon, J.A. (2002). Signals in macaque striate cortical neurons that support the perception of glass patterns. Journal of Neuroscience 22, 8334-8345.

Swanson, W.H. \& BiRch, E.E. (1990). Infant spatiotemporal vision: Dependence of spatial contrast sensitivity on temporal frequency. Vision Research 30, 1033-1048.

Teller, D.Y., Lindsey, D.T., Mar, C.M., Succop, A. \& Mahal, M.R. (1992). Infant temporal contrast sensitivity at low temporal frequencies. Vision Research 32, 1157-1162.

Volkman, F.C. \& Dobson, M.V. (1976). Infant responses of ocular 
fixation to moving visual stimuli. Journal of Experimental Child Psychology 22, 86-99.

Wattam-Bell, J. (1991). Development of motion-specific cortical responses in infancy. Vision Research 31, 287-297.

Wattam-Bell, J. (1992). Development of maximum displacement limits for discrimination of motion direction in infancy. Vision Research 32, $621-630$.
Wattam-Bell, J. (1994). Coherence thresholds for discrimination of motion direction in infants. Vision Research 34, 887-883.

Wattam-Bell, J. (1996). Development of visual motion processing. In Infant Vision, ed. Vital-Durand, F., AtKinson, J. \& Braddick, O., pp. 79-94. Oxford: Oxford University Press.

Wilson, H.R. \& BERGEN, J.R. (1979). A four mechanism model for threshold spatial vision. Vision Research 19, 19-32. 